\title{
Darboux type properties of the paratingent
}

\begin{abstract}
In this paper we consider the Darboux type properties for the paratingent. We review some of the standard facts on the multivalued functions and the paratingent. We prove that the paratingent has always the Darboux property but the property $D_{*}$ holds only when the paratingent is a multivalued function.
\end{abstract}

1. Introduction. It is well known that any continuous real function $f$ : $I \rightarrow \mathbb{R}$, where $I$ is an interval of $\mathbb{R}$ has the Darboux property. This means that if $t<s$ are points in $I$ and $\xi$ is a value between $f(t)$ and $f(s)$ then there exists $x \in[t, s]$ such that $f(x)=\xi$. Moreover, if a function $f$ is differentiable on $I$, then its derivative $f^{\prime}$ has the Darboux property. In [1] J. Czarnowska, G. Kwiecińska consider a generalization of this property for multivalued functions. They define two properties $D$ and $D_{*}$. Both of them are equivalent in the case of a single valued real function but they are not in the case of a mulivalued function. If a function is non-differentiable we can deal with a paratingent instead of a derivative. If a function is locally Lipschitz then the paratingent is a multivalued function on $I$ the values of which are intervals contained in $\mathbb{R}$. In this paper we study conditions under which paratingent enjoys properties $D$ and $D_{*}$. We prove that the

2000 Mathematics Subject Classification. 26E25, 54C60.

Key words and phrases. Paratingent, Darboux property, multivalued functions. 
paratingent always has the property $D$. The property $D_{*}$ is a necessary but not a sufficient condition of continuity of the paratingent.

2. Notation and definitions. Let $\mathbb{R}$ be the set of all real numbers. Let $I \subset \mathbb{R}$ be an interval. We denote by $P(\mathbb{R})$ the family of all nonempty subsets of $\mathbb{R}, C(\mathbb{R})$ the family of all nonempty and closed subsets of $\mathbb{R}$ and Conv $\mathbb{R}$ the family of compact intervals of $\mathbb{R}$.

Definition 2.1. A mapping $F: A \rightarrow P(\mathbb{R})$, where $A$ is a nonempty subset of $\mathbb{R}$, is called a multivalued function.

For a multivalued function $F: I \rightarrow P(\mathbb{R})$ and any $A \subset I$ and $B \subset \mathbb{R}$, we denote

$$
\begin{aligned}
F(A) & =\bigcup\{F(x): x \in A\}, \\
F^{+}(B) & =\{x \in I: F(x) \subset B\}, \\
F^{-}(B) & =\{x \in I: F(x) \cap B \neq \emptyset\} .
\end{aligned}
$$

Definition 2.2. A multivalued function $F: I \rightarrow P(\mathbb{R})$ is lower semicontinuous if for every open set $V \subset \mathbb{R}$ the set $F^{-}(V)$ is open in $I$.

Definition 2.3. A multivalued function $F: I \rightarrow P(\mathbb{R})$ is upper semicontinuous if for every open set $V \subset \mathbb{R}$ the set $F^{+}(V)$ is open in $I$.

Definition 2.4. A multivalued function $F: I \rightarrow P(\mathbb{R})$ is continuous if it is both lower and upper semicontinuous.

In [2] we can find an equivalent condition of lower semicontinuity of multivalued functions.

Theorem 2.5. A multifunction $F: I \rightarrow P(\mathbb{R})$ is lower semicontinuous if and only if

$$
\forall\left\{t_{n}\right\} \subset I, t_{n} \rightarrow t \forall_{x \in F(t)} \exists_{\left\{x_{n}\right\} \subset \mathbb{R}, x_{n} \rightarrow x} \exists_{n_{0}} \forall_{n \geq n_{0}} x_{n} \in F\left(t_{n}\right)
$$

(see [2, Remark 2, p. 10]).

The following theorem gives the condition which implies upper semicontinuity:

Theorem 2.6. If a multifunction $F: I \rightarrow P(\mathbb{R})$ satisfies the condition:

$$
\forall_{\left\{t_{n}\right\} \subset I, t_{n} \rightarrow t} \forall_{x_{n} \in F\left(t_{n}\right)} \exists_{\left\{x_{n_{k}}\right\} \subset\left\{x_{n}\right\}} \lim _{k \rightarrow \infty} x_{n_{k}}=x \in F(t)
$$

then $F$ is upper semicontinuous.

Proof. Assume that

$$
\forall_{\left\{t_{n}\right\} \subset I, t_{n} \rightarrow t} \forall_{x_{n} \in F\left(t_{n}\right)} \exists_{\left\{x_{n_{k}}\right\} \subset\left\{x_{n}\right\}} \lim _{k \rightarrow \infty} x_{n_{k}}=x \in F(t)
$$

and $F$ is not upper semicontinuous. Then there exists open set $G \subset \mathbb{R}$ such that $F^{+}(G)$ is not open in $I$. It follows that there is $a \in F^{+}(G)$, which is not 
an interior point of $F^{+}(G)$. Since $F(a) \subset G$, we have $F(a) \cap(\mathbb{R} \backslash G)=\emptyset$. Because $a$ is not an interior point of $F^{+}(G), K\left(a, \frac{1}{n}\right)$ is not included in $F^{+}(G)$ for all $n \in \mathbb{N}$ where $K(a, \varepsilon)=\{t \in I:|t-a| \leq \varepsilon\}$. Thus there exists a sequence $\left\{t_{n}\right\} \subset I$ such that $t_{n} \in K\left(a, \frac{1}{n}\right)$ and $t_{n} \notin F^{+}(G)$. This implies respectively that $\lim _{n \rightarrow \infty} t_{n}=a$ and $F\left(t_{n}\right)$ is not included in $G$. It follows that there exists a sequence $\left\{x_{n}\right\}$ such that $x_{n} \in\left(F\left(t_{n}\right) \backslash G\right) \subset(\mathbb{R} \backslash G)$. The set $\mathbb{R} \backslash G$ is closed. Therefore the limit of every convergent subsequence of $\left\{x_{n}\right\}$ belongs to $\mathbb{R} \backslash G$ so does not belong to $G$. This contradiction proves that $F$ is upper semicontinuous.

Proposition 2.7. If $F: I \rightarrow P(\mathbb{R})$ has connected values and is upper semicontinuous or lower semicontinuous, then for every connected $C \subseteq I$, $F(C)$ is connected.

The proof of this proposition can be found in [3, Prop. 2.24, p. 43-44].

Definition 2.8. A function $\varphi: I \rightarrow \mathbb{R}$ is said to be locally Lipschitz at $t_{0}$ if

$$
\exists_{L>0} \exists_{\delta>0} \forall\left|t-t_{0}\right|<\delta,\left|s-t_{0}\right|<\delta|\varphi(t)-\varphi(s)| \leq L|t-s| .
$$

In [1] the following definitions of the Darboux properties for multivalued functions were given:

Definition 2.9. A multivalued function $F: I \rightarrow P(\mathbb{R})$ has property $D$ if the image $F(C)$ is connected for any connected set $C \subset I$.

Definition 2.10. A multivalued function $F: I \rightarrow P(\mathbb{R})$ has property $D_{*}$ if for any points $t_{1}, t_{2} \in I, t_{1}<t_{2}$ and every $x_{1} \in F\left(t_{1}\right)$ there exists $x_{2} \in F\left(t_{2}\right)$ such that $\left(\min \left\{x_{1}, x_{2}\right\}, \max \left\{x_{1}, x_{2}\right\}\right) \subset F\left(\left(t_{1}, t_{2}\right)\right)$.

As we know [1] both properties $D$ and $D_{*}$ are equivalent to the Darboux property in the case of a single valued function. However, the class of multivalued functions with the property $D$ is greater in the sense of inclusion than the class of multivalued functions with connected values and property $D_{*}$.

The following theorems are true:

Theorem 2.11. Let $F: I \rightarrow P(\mathbb{R})$ be a multivalued function such that $F(t)$ is a connected set for each $t \in I$. If $F$ has property $D_{*}$ then it has property D.

Theorem 2.12. If a mulivalued function $F: I \rightarrow C(\mathbb{R})$ is continuous then $F$ has property $D_{*}$.

The proofs of Theorem 2.11 and Theorem 2.12 can be found in [1]. Now we introduce the following definition: 
Definition 2.13. Let $\varphi: I \rightarrow \mathbb{R}$ be a continuous function and $t \in I$. The set defined by

$$
\begin{aligned}
&(P \varphi)(t):=\{x \in \mathbb{R}: \exists_{\left\{t_{n}\right\},\left\{s_{n}\right\} \subset I}\left(t_{n} \neq s_{n}, n=1,2, \ldots\right. \\
&\left.\left.\wedge \lim _{n \rightarrow \infty} t_{n}=\lim _{n \rightarrow \infty} s_{n}=t\right) \Rightarrow\left(x=\lim _{n \rightarrow \infty} \frac{\varphi\left(t_{n}\right)-\varphi\left(s_{n}\right)}{t_{n}-s_{n}}\right)\right\}
\end{aligned}
$$

is said to be the paratingent of $\varphi$ at point $t$.

Remark 2.14. The paratingent $(P \varphi)(t)$ at some $t \in I$ can be empty, bounded or unbounded set.

\section{The paratingent of the function.}

Definition 3.1. If for every $t \in I$ the set $(P \varphi)(t)$ is nonempty then the multivalued function $P \varphi: I \rightarrow P(\mathbb{R})$ defined by

$$
I \ni t \rightarrow(P \varphi)(t) \in P(\mathbb{R})
$$

is called the paratingent of function $\varphi$ on $I$.

Remark 3.2. If a function $\varphi: I \rightarrow \mathbb{R}$ is locally Lipschitz then $(P \varphi)(t) \neq \emptyset$, $t \in I$. Indeed, quotients $\left|\frac{\varphi(\tau)-\varphi(\sigma)}{\tau-\sigma}\right|$ are equi-bounded for $\tau$ and $\sigma$ sufficiently close to $t$.

The following properties hold:

Proposition 3.3. The paratingent $(P \varphi)(t)$ is a closed set for all $t \in I$.

Proof. Let $t \in I, x_{n} \in(P \varphi)(t), n=1,2, \ldots$ and $\lim _{n \rightarrow \infty} x_{n}=x, x \in \mathbb{R}$. We have

$$
x_{n}=\lim _{i \rightarrow \infty} \frac{\varphi\left(t_{i}^{n}\right)-\varphi\left(s_{i}^{n}\right)}{t_{i}^{n}-s_{i}^{n}}
$$

where $t_{i}^{n}, s_{i}^{n} \in I, t_{i}^{n} \neq s_{i}^{n}$ and $\lim _{i \rightarrow \infty} t_{i}^{n}=\lim _{i \rightarrow \infty} s_{i}^{n}=t, n, i=1,2, \ldots$. Then for every $n$ there exists such $i_{n}$ that

$$
\left|t_{i_{n}}^{n}-t\right|<\frac{1}{n}, \quad\left|s_{i_{n}}^{n}-t\right|<\frac{1}{n} \quad \text { and } \quad\left|\frac{\varphi\left(t_{i_{n}}^{n}\right)-\varphi\left(s_{i_{n}}^{n}\right)}{t_{i_{n}}^{n}-s_{i_{n}}^{n}}-x_{n}\right|<\frac{1}{n} .
$$

Hence

$$
x=\lim _{n \rightarrow \infty} \frac{\varphi\left(t_{i_{n}}^{n}\right)-\varphi\left(s_{i_{n}}^{n}\right)}{t_{i_{n}}^{n}-s_{i_{n}}^{n}}
$$

where $\lim _{n \rightarrow \infty} t_{i_{n}}^{n}=\lim _{n \rightarrow \infty} s_{i_{n}}^{n}=t$. So $x \in(P \varphi)(t)$. This means that $(P \varphi)(t)$ is a closed set.

Lemma 3.4. Let $x, y \in(P \varphi)(t), t \in I$. If $x<z<y$ then $z \in(P \varphi)(t)$. 
Proof. Let $x<z<y, x \in(P \varphi)(t)$ and $y \in(P \varphi)(t), t \in I$. Then

$$
\begin{array}{r}
\exists\left\{t_{n}\right\},\left\{s_{n}\right\} \subset I\left(t_{n} \neq s_{n}, n=1,2, \ldots \wedge \lim _{n \rightarrow \infty} t_{n}=\lim _{n \rightarrow \infty} s_{n}=t\right) \\
\Rightarrow\left(x=\lim _{n \rightarrow \infty} \frac{\varphi\left(t_{n}\right)-\varphi\left(s_{n}\right)}{t_{n}-s_{n}}\right)
\end{array}
$$

and

$$
\begin{array}{r}
\exists_{\left\{\tau_{n}\right\},\left\{\sigma_{n}\right\} \subset I}\left(\tau_{n} \neq \sigma_{n}, n=1,2, \ldots \wedge \lim _{n \rightarrow \infty} \tau_{n}=\lim _{n \rightarrow \infty} \sigma_{n}=t\right) \\
\Rightarrow\left(y=\lim _{n \rightarrow \infty} \frac{\varphi\left(\tau_{n}\right)-\varphi\left(\sigma_{n}\right)}{\tau_{n}-\sigma_{n}}\right) .
\end{array}
$$

We can assume that $t_{n}<s_{n}$ and $\tau_{n}<\sigma_{n}$.

Let $\alpha \in[0,1]$. We define

$$
u_{n}^{\alpha}=(1-\alpha) t_{n}+\alpha \tau_{n}, \quad v_{n}^{\alpha}=(1-\alpha) s_{n}+\alpha \sigma_{n} .
$$

We see that $\lim _{n \rightarrow \infty} u_{n}^{\alpha}=\lim _{n \rightarrow \infty} v_{n}^{\alpha}=t$. Moreover,

$$
\begin{aligned}
\min \left\{t_{n}, \tau_{n}\right\} & \leq u_{n}^{\alpha} \leq \max \left\{t_{n}, \tau_{n}\right\}, \\
\min \left\{s_{n}, \sigma_{n}\right\} & \leq v_{n}^{\alpha} \leq \max \left\{s_{n}, \sigma_{n}\right\}
\end{aligned}
$$

and

$$
u_{n}^{\alpha}<v_{n}^{\alpha}
$$

Let $g_{n}: I \rightarrow \mathbb{R}$ be given by

$$
g_{n}(\alpha)=\frac{\varphi\left(u_{n}^{\alpha}\right)-\varphi\left(v_{n}^{\alpha}\right)}{u_{n}^{\alpha}-v_{n}^{\alpha}} .
$$

Let

$$
x_{n}=\frac{\varphi\left(t_{n}\right)-\varphi\left(s_{n}\right)}{t_{n}-s_{n}}, \quad y_{n}=\frac{\varphi\left(\tau_{n}\right)-\varphi\left(\sigma_{n}\right)}{\tau_{n}-\sigma_{n}} .
$$

We have $g_{n}(0)=x_{n}$ and $g_{n}(1)=y_{n}$.

Let $z_{n} \in I$ be such that $x_{n}<z_{n}<y_{n}$ and $\lim _{n \rightarrow \infty} z_{n}=z$. In view of Darboux property for real function for every $n \in \mathbb{N}$ there exists $\alpha_{n}$ such that:

$$
g_{n}\left(\alpha_{n}\right)=\frac{\varphi\left(u_{n}^{\alpha_{n}}\right)-\varphi\left(v_{n}^{\alpha_{n}}\right)}{u_{n}^{\alpha_{n}}-v_{n}^{\alpha_{n}}}=z_{n} .
$$

Observe, that (3.1) implies $\lim _{n \rightarrow \infty} u_{n}^{\alpha_{n}}=\lim _{n \rightarrow \infty} v_{n}^{\alpha_{n}}=t$. Hence

$$
z=\lim _{n \rightarrow \infty} z_{n}=\frac{\varphi\left(u_{n}^{\alpha_{n}}\right)-\varphi\left(v_{n}^{\alpha_{n}}\right)}{u_{n}^{\alpha_{n}}-v_{n}^{\alpha_{n}}} \in(P \varphi)(t), t \in I .
$$

Lemma 3.5. The subset $A \subset \mathbb{R}$ is connected if and only if the condition $(x \in A, y \in A, x<z<y \Rightarrow z \in A)$ holds.

For a proof see [4, Theorem 2.47]. The next proposition follows from Lemma 3.4 and Lemma 3.5: 
Proposition 3.6. The paratingent $(P \varphi)(t)$ is connected for all $t \in I$.

Proposition 3.7. Let $\varphi: I \rightarrow \mathbb{R}$ be locally Lipschitz. Then the paratingent $P \varphi: I \rightarrow P(\mathbb{R})$ is the upper semicontinuous multivalued function.

Proof. Let $\left\{a_{n}\right\} \in I, a \in I$ and $\lim _{n \rightarrow \infty} a_{n}=a$. Moreover let $\left\{x_{n}\right\} \in$ $(P \varphi)\left(a_{n}\right), n=1,2, \ldots$ Then

$$
\begin{array}{r}
\exists\left\{t_{i}^{n}\right\},\left\{s_{i}^{n}\right\} \subset I \\
\quad\left(t_{i}^{n} \neq s_{i}^{n}, n=1,2, \ldots \wedge \lim _{i \rightarrow \infty} t_{i}^{n}=\lim _{i \rightarrow \infty} s_{i}^{n}=a_{n}\right) \\
\Rightarrow\left(x_{n}=\lim _{i \rightarrow \infty} \frac{\varphi\left(t_{i}^{n}\right)-\varphi\left(s_{i}^{n}\right)}{t_{i}^{n}-s_{i}^{n}}\right), i, n=1,2, \ldots
\end{array}
$$

There exist $L>0$ and $\delta>0$ such that the function $\varphi$ satisfies the Lipschitz condition with constant $L$ in the interval $(a-\delta, a+\delta) \cap I$. For every fixed $n \in \mathbb{N}$ there exists an index $m_{n}$ such that $\left|a_{m_{n}}-a\right|<\frac{1}{n}$ and

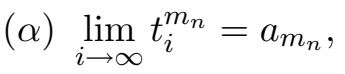

$$
\begin{aligned}
& \text { (ß) } \lim _{i \rightarrow \infty} s_{i}^{m_{n}}=a_{m_{n}} \text {, } \\
& (\gamma) \lim _{i \rightarrow \infty} \frac{\varphi\left(t_{i}^{m_{n}}\right)-\varphi\left(s_{i}^{m_{n}}\right)}{t_{i}^{m_{n}}-s_{i}^{m_{n}}}=x_{m_{n}}
\end{aligned}
$$

where $t_{i}^{m_{n}} \neq s_{i}^{m_{n}}, i=1,2, \ldots$ The conditions $(\alpha),(\beta)$ and $(\gamma)$ imply

$$
\begin{array}{lll}
\exists_{j_{n} \in \mathbb{N}} & \forall_{i \geq j_{n}} & \left|t_{i}^{m_{n}}-a_{m_{n}}\right|<\frac{\delta}{2 n}, \\
\exists_{k_{n} \in \mathbb{N}} & \forall_{i \geq k_{n}} & \left|s_{i}^{m_{n}}-a_{m_{n}}\right|<\frac{\delta}{2 n}, \\
\exists_{l_{n} \in \mathbb{N}} & \forall_{i \geq l_{n}} & \left|\frac{\varphi\left(t_{i}^{m_{n}}\right)-\varphi\left(s_{i}^{m_{n}}\right)}{t_{i}^{m_{n}}-s_{i}^{m_{n}}}-x_{m_{n}}\right|<\frac{1}{n} .
\end{array}
$$

Particularly for $i_{n}=\max \left(j_{n}, k_{n}, l_{n}\right)$ we have

$$
\begin{aligned}
& \left|t_{i_{n}}^{m_{n}}-a_{m_{n}}\right|<\frac{\delta}{2 n}, \\
& \left|s_{i_{n}}^{m_{n}}-a_{m_{n}}\right|<\frac{\delta}{2 n}, \\
& \left|\frac{\varphi\left(t_{i_{n}}^{m_{n}}\right)-\varphi\left(s_{i_{n}}^{m_{n}}\right)}{t_{i_{n}}^{m_{n}}-s_{i_{n}}^{m_{n}}}-x_{m_{n}}\right|<\frac{1}{n} .
\end{aligned}
$$

Since absolute values of quotients $\left(\varphi\left(t_{i_{n}}^{m_{n}}\right)-\varphi\left(s_{i_{n}}^{m_{n}}\right)\right) /\left(t_{i_{n}}^{m_{n}}-s_{i_{n}}^{m_{n}}\right)$ are bounded by $L$, we can choose a sequence $\left(n_{k}\right)$ such that

$$
\frac{\varphi\left(t_{i_{n_{k}}}^{m_{n_{k}}}\right)-\varphi\left(s_{i_{n_{k}}}^{m_{n_{k}}}\right)}{t_{i_{n_{k}}}^{m_{n_{k}}}-s_{i_{n_{k}}}^{m_{n_{k}}}}
$$


is convergent to $x \in \mathbb{R}$. Moreover from $\lim _{k \rightarrow \infty} t_{i_{n_{k}}}^{m_{n_{k}}}=\lim _{k \rightarrow \infty} s_{i_{n_{k}}}^{m_{n_{k}}}=a$ we obtain $x \in(P \varphi)(a)$. Hence, the upper semicontinuity the paratingent $P \varphi$ follows from Theorem 2.6.

The next theorem is an immediate consequence of Proposition 2.7.

Theorem 3.8. The paratingent $P \varphi$ has property $D$.

Generally the paratingent does not have property $D_{*}$.

Example 3.9. Let $\varphi(t)=|t|, t \in[-1,1]$. The paratingent of $\varphi$ defined by

$$
(P \varphi)(t)= \begin{cases}\{-1\}, & -1 \leq t<0 \\ {[-1,1],} & t=0 \\ \{1\}, & 0<t \leq 1\end{cases}
$$

does not have property $D_{*}$.

Indeed, let $t_{1}=-1, t_{2}=0, x_{1}=-1$. Then we have $(P \varphi)\left(t_{1}\right)=\{-1\}$, $(P \varphi)\left(t_{2}\right)=[-1,1]$ and for all $x_{2} \in(P \varphi)\left(t_{2}\right)$ the interval $\left(x_{1}, x_{2}\right)$ is not included in $(P \varphi)\left(\left(t_{1}, t_{2}\right)\right)=\{-1\}$.

Remark 3.10. We see that if the paratingent $P \varphi$ has property $D_{*}$ then according to Theorem 2.11 it has property $D$ as well.

The following theorem is an immediate consequence of Theorem 2.12.

Theorem 3.11. If a paratingent $P \varphi: I \rightarrow C(\mathbb{R})$ is continuous then $P \varphi$ has property $D_{*}$.

Remark 3.12. If $F: I \rightarrow$ Conv $\mathbb{R}$ is a continuous multivalued function then there exists a function $\varphi: I \rightarrow \mathbb{R}$ such that $(P \varphi)(t)=F(t)$ (see [5]).

The converse of Theorem 3.11 is not true. For example let us consider the function

$$
\varphi(t)= \begin{cases}0, & t=0, \\ (-1)^{n}\left[2^{n+1}\left(t-\frac{3}{2^{n+2}}\right)^{2}-\frac{1}{2^{n+3}}\right], & t \in\left(\frac{1}{2^{n+1}}, \frac{1}{2^{n}}\right], n=0,1,2, \ldots\end{cases}
$$

Then

$$
(P \varphi)(t)= \begin{cases}{[-1,1],} & t=0, \\ \left\{\varphi^{\prime}(t)\right\}, & t \in\left(\frac{1}{2^{n+1}}, \frac{1}{2^{n}}\right], \quad n=0,1,2, \ldots .\end{cases}
$$

where $\varphi^{\prime}(t)=(-1)^{n}\left(2^{n+2} t-3\right), t \in\left(\frac{1}{2^{n+1}}, \frac{1}{2^{n}}\right], n=0,1,2, \ldots$.

The paratingent $P \varphi$ has property $D_{*}$ but it is not lower semicontinuous. To see this let us consider the sequence

$$
t_{n}=\frac{3}{2^{n+2}}, n=0,1,2, \ldots
$$

Of course $\lim _{n \rightarrow \infty} t_{n}=0$ and $(P \varphi)\left(t_{n}\right)=\{0\}$. Let $x=1$ and let $\left\{x_{n}\right\}$ be any sequence convergent to $x$. Then for $n \geq \bar{n}$ we have $x_{n}>0$. Hence 
$x_{n} \notin(P \varphi)\left(t_{n}\right)=\{0\}$ for $n \geq \bar{n}$. This means that the paratingent is not lower semicontinuous so it is not continuous.

\section{REFERENCES}

[1] Czarnowska, J., Kwiecińska, G., On the Darboux property of mulivalued functions, Demonstratio Math. 25 (1992), 193-199.

[2] Delahaye, J. P., Denel, J., The continuites of the point-to-set maps, definitions and equivalences, Math. Programming Stud. 10 (1979), 8-12.

[3] Hu, S., Papageorgiou, N. S., Handbook of Multivalued Analysis. Vol. I. Theory, Kluwer Academic Publisher, Dordrecht, 1997.

[4] Rudin, W., Principles of Mathematical Analysis, Third edition, McGraw-Hill Book Co., New York-Auckland-Düsseldorf, 1976.

[5] Zygmunt, W., On the full solution of the paratingent equations, Ann. Univ. Mariae Curie-Skłodowska Sect. A 26 (1972), 103-108 (1974).

Małgorzata Fedor

Department of Real Analysis

Faculty of Mathematics and Natural Sciences

The John Paul II Catholic University of Lublin

ul. Konstantynów $1 \mathrm{H}$

20-950 Lublin, Poland

e-mail: mfedor@kul.lublin.pl

Joanna Szyszkowska

Department of Real Analysis

Faculty of Mathematics and Natural Sciences

The John Paul II Catholic University of Lublin

ul. Konstantynów 1H

20-950 Lublin, Poland

e-mail: jszysz@kul.lublin.pl

Received February 7, 2008 